\title{
Dental Age Assessment: a comparison of 4- to 24-year-olds in the United Kingdom and an Australian population
}

\author{
TANYA SHYAMI PEIRIS', GRAHAM J. ROBERTS ${ }^{2}$ \& NEETA PRABHU ${ }^{1}$ \\ ${ }^{1}$ Department of Paediatric Dentistry, Westmead Centre for Oral Health, Sydney, NSW 2145 Australia, and ${ }^{2}$ Dental Paediatrics, \\ King's College London Dental Institute, Bessemer Road, London SE5 9RS, UK
}

International Journal of Paediatric Dentistry 2009

Background. The physiological age of a person is determined by the degree of maturation of the different tissue systems. Children of the same chronological age (CA) can demonstrate different degrees of maturation. Dental age (DA) is based on the maturation of teeth. Tooth formation is a continuous process, where the developmental stages of the tooth can be sequenced and defined depending on the degree of mineralization. These stages can be visualized on a dental panoramic tomograph (DPT).

Aim. The aim of this study was to use a new method of Dental Age Assessment (DAA) to compare a United Kingdom (UK) and an Australian (AUS) population.

Design. The DPTs used are from the archives of the Westmead Centre for Oral Health (Westmead, Australia) and the King's College London Dental Insti- tute. From the preliminary sample of 89 DPTs from each population, 77 were suitable for use as matched pairs. The radiographic technique used was developed by Demirjian and describes eight stages of tooth development. This was used in combination with numerical data derived from a metaanalysis of a single UK subject.

Results. A significant difference was shown between the CA and DA of the AUS patients. The AUS patients were also shown to have a significant 0.82 years delay in their DA compared to the UK patients. The findings indicate a difference in AUS compared to UK patients. These results indicate the need to develop a reference data set for the AUS population for DAA.

Conclusions. This research is of significance in a number of clinical disciplines and can also be used to assist in age determination of subjects of unknown birth date to assist in forensic dentistry or social deliberations.

\section{Introduction}

\section{The concept of 'age'}

The physiological age of a person is determined by the degree of maturation of the different tissue systems ${ }^{1}$. Physiological age can be used to define a child's 'progress towards completeness of development or maturity ${ }^{2}$. The result can be best expressed in terms of the individual system studied. Within a tissue system, the sequence of one or more irreversible events defines maturation ${ }^{2}$.

Children of the same chronological age (CA) can demonstrate different degrees of matura-

\section{Correspondence to:}

Neeta Prabhu, Department of Paediatric Dentistry, Level 3, Westmead Centre for Oral Health, Darcy Road, Westmead, NSW 2145 Australia.

E-mail:neeta_prabhu@wsahs.nsw.gov.au tion $^{3}$. Physiological age can be subdivided into a number of biological categories including skeletal age, morphological age, secondary sex characteristic age, and dental age $(\mathrm{DA})^{1}$. DA is usually based on the maturation of teeth. The different biological ages can be used as a single entity or combined to assess the maturation of a growing child. DA and CA are two indicators of biological maturity which collectively have the least variability ${ }^{4}$.

Determination of age is of importance in forensic medicine, paediatric endocrinology, and treatment planning in orthodontics and paediatric dentistry ${ }^{5}$.

\section{$D A$}

Tooth formation is a continuous process, where the developmental stages of the tooth can be sequenced and defined ${ }^{6}$. The stages of tooth formation are not of equal space or duration during growth ${ }^{7}$. Each tooth goes through the 
same stages in every individual, and although each stage is indicative of maturation it is not related to size ${ }^{1}$.

\section{Dental age assessment (DAA)}

This can be quantified using a number of methods including crown root measurements, time of emergence of the crown in the mouth, and radiographic analysis of tooth formation. A number of studies have been carried out to identify the most appropriate and precise method to estimate DA. The DAA method which gives a result closest to the gold standard of CA is the best method.

\section{Tooth development stages (TDS)}

The radiographic method of DAA is based on the degree of tooth mineralization. It uses up to 16 tooth morphology types and can be used in children up to 21 years of age. Analysis is twofold; firstly, it involves observation of the TDS present on a radiograph and secondly, using a predetermined scale to score the teeth. The scores are added up to give a corresponding DA. The TDS as described by Demirjian et al. ${ }^{1}$ can be visualized on a dental panoramic tomograph (DPT).

\section{Radiographic assessment of DAA}

The radiographic appearance of the teeth to estimate age was first assessed in $1960^{8}$. This method was found to have low precision with regard to accuracy ${ }^{7}$. Further investigation in 1973 resulted in the creation of a dental maturity scaling system valid for universal use ${ }^{1}$. A number of other radiographic DAA methods exist which identify different numbers of TDS $^{9-12}$; however, Demirjian's method has been found to be the most accurate ${ }^{13}$.

\section{Demirjian's DAA method}

Demirjian's method is based on the observation of seven mandibular teeth in children of French Canadian origin aged 3-17 years. Eight stages of mineralization were identified for each tooth. 'Line drawings and radiographic illustrations $^{\prime 14}$ were used to represent each stage
Stages were allocated a score ${ }^{15}$. In the second part of the method, the sum of the scores was used to estimate DA on a scale of 0-100. Boys and girls were given different scales. This method has been deemed easy to use ${ }^{7}$ having 'clear descriptive criteria'14 for assessing the TDS.

Demirjian's 'full' method only takes us up to 15.5 years, when the lower left second molar (LL7) completes growth. This artificially limits dental maturity to 16 years of age. The difficulty with this method is that the third molars are excluded. To overcome this obstacle, investigators have used the first part of Demirjian's method that is the precise anatomical descriptions of the TDS and applied this to third molars. Hence, increasing the age this method can be used to up to 21 years.

This DAA method was found to have such high accuracy that the method was recommended to evaluate mineralization of third molars in forensic age determination investigations $^{13}$.

Even though Demirjian's full method has proved universal in application, some researchers believe when converting the score to DA, the specific population must be considered ${ }^{16}$. When analysing South Indian children, it was hypothesized that different populations should be assessed using scales that were devised on the same population group to ensure accuracy ${ }^{5}$.

Others also believe the younger a child is, the more accurate the estimation of age $e^{5}$. This is because of the faster rate of development and greater number of developing teeth present ${ }^{17}$. If DA is to be used to estimate age, other indicators of biological maturity must also be used to improve accuracy ${ }^{6}$.

\section{Culturally diverse populations}

The Demirjian method has not been extensively studied in an Australian (AUS) population. A previous study on South Australian TDS of children aged 5-16 years of age was analysed in seven mandibular teeth on a DPT. The French Canadian standards were then used to estimate the DA of the subjects. The estimated DA and CA were then compared. The 'estimated DA coincided with CA in only a small percentage' of subjects ${ }^{18} ; 34.7 \%$ of 
boys and $36.9 \%$ of girls had the estimated DA and CA within 0.5 years. A difference greater than 0.5 years was said to be not suitable for forensic age determination. The South Australian children were found to be less dentally mature in the younger age groups, but more advanced over the age of 15 years compared to the French Canadian children ${ }^{18}$. This portrays a frequent overestimation of CA in the younger age groups when using the French Canadian standards.

Another study carried out on children in Western Australia (Perth) determined dental maturity by analysis of four permanent mandibular teeth. The French Canadian standards determined by Demirjian were used to evaluate the dental maturation of the Perth children. Girls were found to be more dentally mature, whereas boys in the older age groups were less dentally mature by up to 12 months ${ }^{19}$.

The United Kingdom (UK) population has been used in a number of DAA studies. A previous study found British children to be more dentally advanced ${ }^{20}$ than Demirjian's French Canadian sample. In the past 25 years, a positive secular trend has been noted that may account for the advancement in dental maturity or age of British children. A meta-analysis of previous retrospective cross-sectional studies using Demirjian's full method to assess dental maturity compared populations from Australia, Belgium, Canada, England, Finland, France, South Korea, and Sweden ${ }^{21}$. No major differences were found in the timing of the TDS, thus failing to provide an explanation found in previous studies ${ }^{7}$.

This study will involve a DAA comparing UK and AUS children aged 4-24 years using a new method $^{22}$. No study to date has compared a UK and AUS population using a combination of Demirjian's anatomical stages ${ }^{1}$ and numerical data derived from a meta-analysis of single UK subjects. The DA and CA of the children will be analysed and compared. The DAA in this study is based on numerical data from the TDS present in a single subject which is then averaged within each stage using the mathematical methods of meta-analysis ${ }^{22}$.

The aim of this study was to use a new method of DAA to compare two culturally diverse populations, which have not been studied previously in this context.

\section{Materials and methods}

\section{Data protection and ethical approval}

The project proposal for this study was approved by the Scientific Advisory Committee and Research Ethics Committee of the University of Sydney and Westmead Hospital, Sydney West Area Health Service. Registration of the project with The Data Protection Officer at King's College Dental Hospital ensured this study complied with Data Protection Legislation.

\section{Radiographs}

This project uses DPTs from the archives of the Westmead Centre for Oral Health (Westmead, Australia) and the King's College London Dental Institute (Guy's, King's College, and St Thomas' Hospitals, London). The DPTs were photographed (Fig. 1) using a Pentax *ist D single lens reflex digital camera. The digital images were stored electronically, each with a unique identity. The AUS and UK subjects each had individual folders containing the digital images.

\section{TDS}

The method for assessing TDS described by Demirjian and Goldstein ${ }^{15}$ was used. This method comprises eight stages of anatomical configurations (Fig. 2). In order to improve the reliability of assessments of the TDS, printed copies of the Demirjian stages were continually referred to by the assessor. In each radiograph, only the upper and lower of the left side were assessed in this study. Note teeth with TDS H that is closed apex were excluded from the study. Stage $\mathrm{H}$ is the only TDS shown to not follow a normal distribution curve ${ }^{21}$ and so is regarded as inaccurate. The data relating to the TDS were entered into a database designed to relate the data to the TDS on the DPTs. The data were entered with the essential demographic details, date of radiograph, missing teeth, and Demirjian data.

\section{Computer software}

Microsoft Excel was utilized to create a workbook. A total of 89 DPT digital images were 

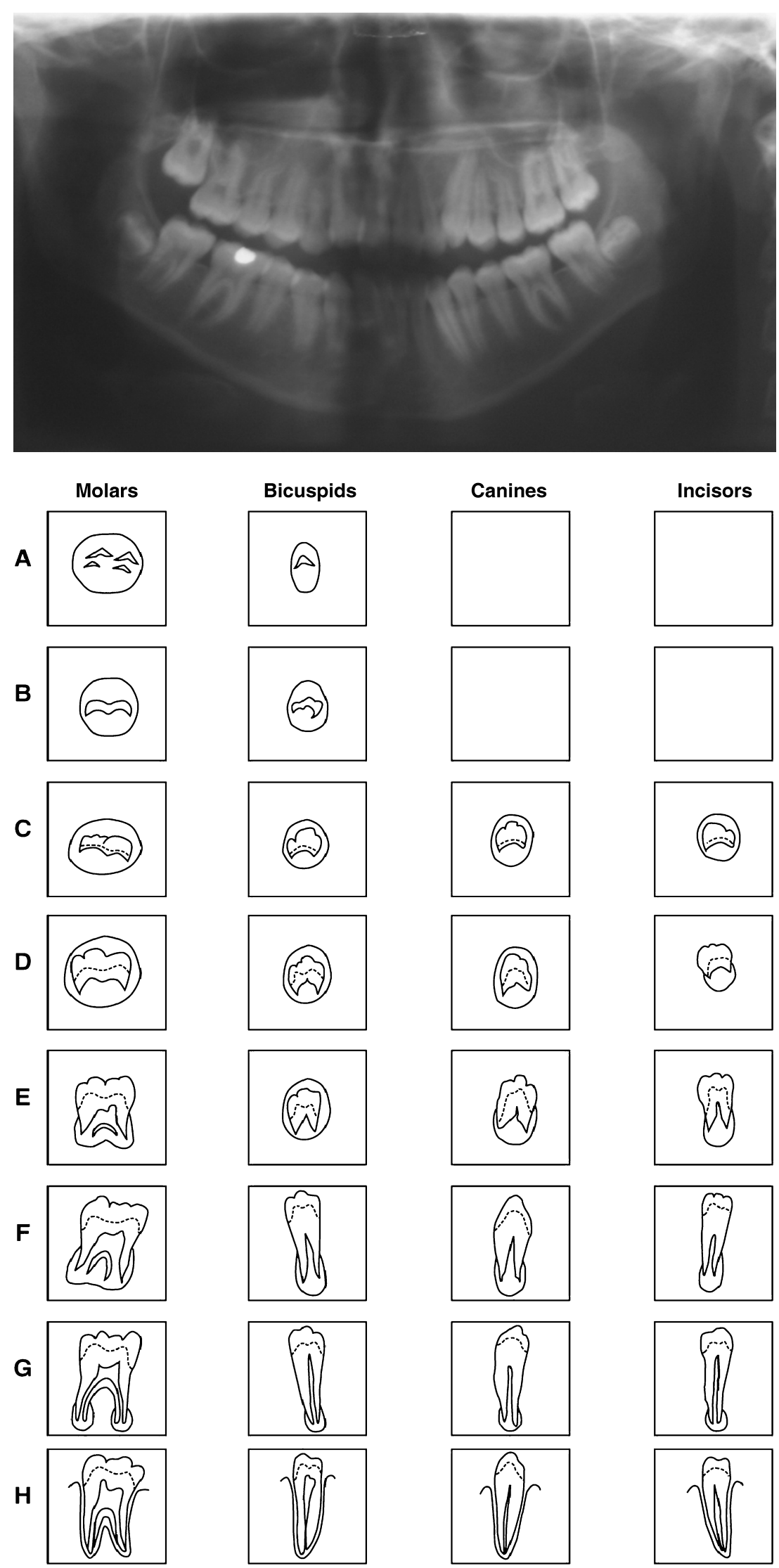

Schematic representation for eight stages of development (after Demirjian 1973)
Fig. 1. An example of a dental panoramic tomograph of an Australian patient.
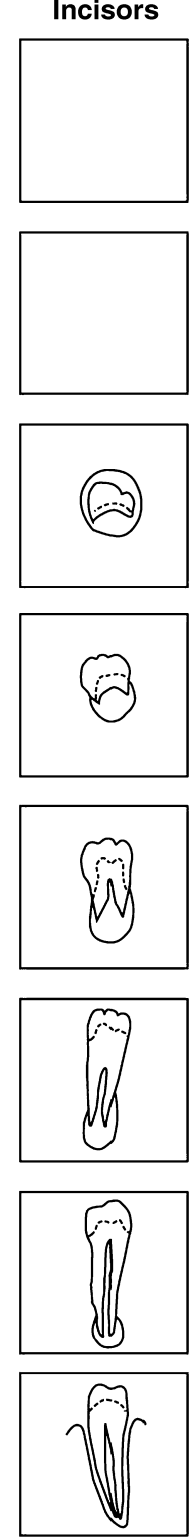

Fig. 2. The eight tooth development stages as described by Demirjian (1973). 
Table 1. Example of summary data (mean and standard error) extracted from Microsoft Excel tables and copied into the data sheet of STATA. The data used in this example are from the subject in Fig. 1.

\begin{tabular}{llcc}
\hline Tooth & Stage & x (Mean) & SE (standard error) \\
\hline Maxillary & & & \\
UL1 & UL1F & 7.17 & 0.20 \\
UL2 & UL2E & 6.20 & 0.19 \\
UL3 & UL3E & 7.27 & 0.20 \\
UL4 & UL4D & 7.15 & 0.15 \\
UL5 & UL5D & 7.27 & 0.15 \\
UL6 & UL6G & 8.07 & 0.16 \\
UL7 & UL7D & 7.71 & 0.12 \\
UL8 & M4 & & 0.12 \\
Mandibular & & & \\
LL1 & LL1F & 6.16 & 0.22 \\
LL2 & LL2E & 6.17 & 0.47 \\
LL3 & LL3E & 6.88 & 0.21 \\
LL4 & LL4D & 6.40 & 0.20 \\
LL5 & LL5D & 7.18 & 0.19 \\
LL6 & LL6G & 8.00 & 0.16 \\
LL7 & LL7D & 7.62 & 0.13 \\
LL8 & & & \\
\hline
\end{tabular}

$M_{4}$, missing tooth due to reaseon 4 .

used for each population. One worksheet was required for each digital image. The digital image of the radiograph was copied into the corresponding worksheet. The summary data, comprised of $n$ (number of teeth contributing to the data for a TDS), $m$ (mean, the average age of attainment of the individual TDS), and SE (standard error, the estimate of the likely variation of estimate of the mean). The $\mathrm{SE}$ is used in the mathematical processes of meta-analysis.

The first table contained the numerical data for the TDS (Table 1). The first column of this table identified the tooth assessed, for example ULl, interpreted as upper left central incisor or LL4 interpreted as lower left first premolar. The second column stated the TDS. The next column documented the value for mean of age of attainment of the TDS, and the fourth column contained the SE for that TDS.

For each TDS, a distribution of ages was constructed using a Microsoft Access Query. Therefore, information comprising summary of up to 112 Demirjian stages was available. A histogram showing the frequency of age distribution for each TDS was also available. A normal distribution curve was superimposed onto the histogram. This information was used to estimate the DA of the subjects in the study. This was achieved by pasting a small table (Table 1) into STATA comprising the TDS UR4G, the mean age for that stage 11.95 years, and the SE -0.26 years, for the estimation of that mean.

The next table (Table 2) documented the statistical analysis results generated by STATA. A forest plot showing the DA of the subject was also generated in STATA, and copied and pasted into the worksheet.

\section{Statistical analysis and considerations}

STATA is a statistical software used for data analysis. STATA version 8 (2003) held the entire data set in this study and was used to manipulate and analyse all data. Reference interval data were tested for normality. The second table from each Microsoft Excel worksheet was copied and pasted into the data editor, and the frame was closed. The columns were tooth, TDS, $m$, and SE, and these data were used to produce the estimated DA.

\section{Identifying appropriate UK controls}

Once the AUS data analysis was complete, Microsoft Access was utilized to generate queries within the database. The purpose was to choose an appropriately matched UK patient for each AUS subject in the study. The subjects were matched based on age and sex. Queries were used to search for suitable subjects plus 0.25 years and minus 0.25 years of the corresponding AUS patient's CA. The most appropriate subject was chosen and the DPT of the patient retrieved from the database.

The date of birth and date of radiograph were used to determine the CA. Next, the values for TDS were entered into the first table, exactly as entered for AUS patients as above. The $m$ and SE values were also entered for each TDS as for the AUS patients. Statistical analysis using the paired $t$-test was then carried out as undertaken for the AUS subjects.

\section{Inclusion criteria}

Four to twenty-four-year-old male and female subjects who already had a DPT in their 
records for clinical purposes were included from each population. Each UK subject was matched for sex and age \pm 0.25 years, with each AUS subject. All teeth on the left-hand side, that is, quadrants two and three, were included in the TDS analysis. TDS A-G were included in the DAA. Stage $\mathrm{H}$ was excluded (as explained in TDS).

\section{Summary table}

When all data had been entered into the Microsoft Excel worksheets, a summary worksheet was created at the start of the workbook. This worksheet comprised for both AUS and UK: identity number, CA, DA, difference. These data were then entered into STATA and a comparison of means was carried out.

Comparisons were made within each group between CA and DA. The CA and DA of the two populations were also compared.

\section{Inter-rater agreement}

The inter-rater agreement demonstrates the consistency in assessment of the investigator (also known as the 'rater'). The assessors undertook a reproducibility test to measure their reliability in assessing the TDS. A random set of 12 DPTs were viewed 1 week apart.

An inter-rater kappa value of 0.9805 agreement was found for the primary examiner in this study (T.S.P.). Any kappa values between 0.81 and 1.00 have an 'almost perfect' agreement ${ }^{23}$, suggesting this assessor's inter-rater agreement was highly reproducible in this study.

\section{Results}

\section{Sample distribution}

Seventy-seven subjects from each population were selected. The inclusion criteria involved subjects aged between 4 and 24 years of the specific population already having a DPT in their records for clinical purposes. Teeth at stage $\mathrm{H}$ were not included in the age assessment. Thus, 154 subjects in total were analysed in the DAA. Ninety were females and 64 were males. No correlation of DAA results was attributed to patient gender.
Table 2. Example of statistical analysis of chronological age (CA), using data from the subject in Fig. 1. In this example, the estimated $C A$ is 7.1 years.

\begin{tabular}{lcccc}
\hline Estimate & $\begin{array}{c}\text { Lower 99\% } \\
\mathrm{Cl}\end{array}$ & $\begin{array}{c}\text { Upper 99\% } \\
\mathrm{Cl}\end{array}$ & $\boldsymbol{z}$ value & $\boldsymbol{P}$ value \\
\hline 7.125 & 6.710 & 7.539 & 44.267 & 0.000 \\
\hline
\end{tabular}

Each subject had a summary data table comprising mean and standard error for TDS analysed (Table 1), STATA output table (Table 2), and STATA meta-analysis of DA (Fig. 3). The confidence intervals used to estimate age correlated to the subjects from each population.

\section{Comparison of $D A$ and $C A$}

Scatter plots were constructed to demonstrate the range of ages. The DA and CA of the UK subjects were found to be similar with no statistically significant difference between the two.

The overall trend observed with the AUS subjects indicated the DA and CA were different. The DA and CA were compared using the paired $t$-test. This difference was statistically significant $(P<0.001)$.

\section{Comparison between the AUS and UK populations}

Scatter plots were constructed to demonstrate comparisons. The CA of the AUS and UK subjects were similar (Fig. 4). The DA of the AUS and UK populations was found to be different (Fig. 5). The AUS population had a 0.82 year delay in their DA compared to the UK population. This difference was compared and was found to be very statistically significant $(P<0.001)$.

\section{Discussion}

This study is a DAA of samples from two different populations. Both populations were assessed individually and then comparisons were made between the two groups. The data used in this study were based on the TDS described by Demirjian et al. ${ }^{1}$ To ensure the reliability and accuracy of the eight stages of the Demirjian system, the DAA in this study was based on numerical data from the TDS present in a single UK subject. These data were 
Fig. 3. Example of output from STATA of meta-analysis of dental age. The data used in this example are from the subject in Fig. 1. This shows the estimated mean age (the box) and $99 \%$ confidence interval of each developing tooth stage. The area of each box is proportional to the number of children in the sample for that tooth stage of development. The combined estimated dental age is indicated by the dotted line and $99 \%$ confidence interval by the limits of the diamond.

\begin{tabular}{ccccccc}
\hline $\begin{array}{c}\text { Patient } \\
\text { ID }\end{array}$ & $\begin{array}{c}\text { Chronological } \\
\text { age }\end{array}$ & $\begin{array}{c}\text { Dental } \\
\text { age }\end{array}$ & $\begin{array}{c}\text { Lower 99\% } \\
\text { confidence } \\
\text { interval }\end{array}$ & $\begin{array}{c}\text { Upper 99\% } \\
\text { confidence } \\
\text { interval }\end{array}$ & $\begin{array}{c}\text { Date of } \\
\text { birth }\end{array}$ & $\begin{array}{c}\text { Date of } \\
\text { radiograph }\end{array}$ \\
\hline ST05_074 & 7.20 & 7.13 & 6.71 & 7.54 & $17 / 6 / 1998$ & $29 / 8 / 2005$ \\
\hline
\end{tabular}

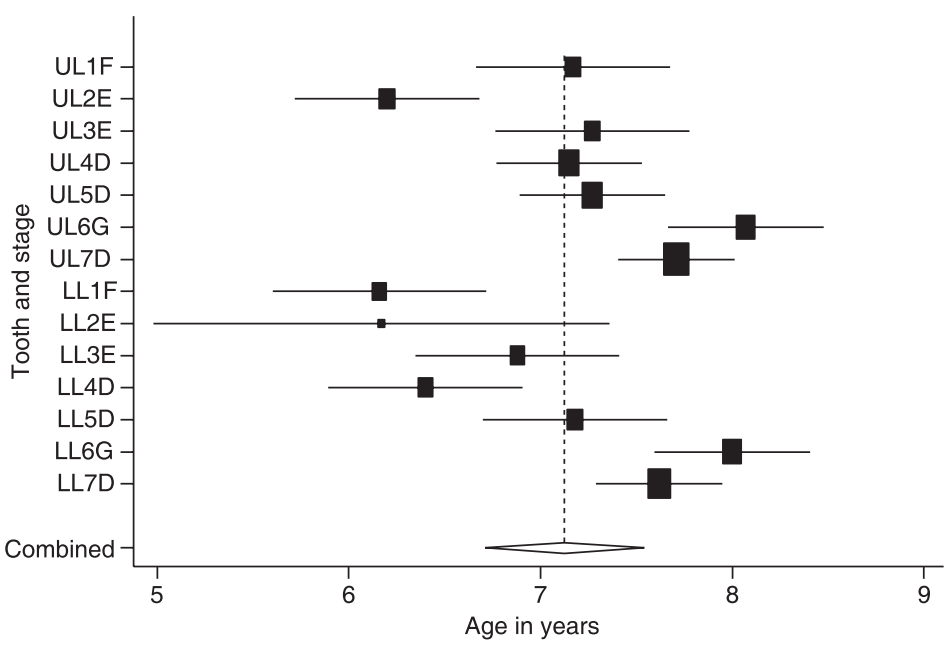

Dental age assessment: ST05_074

Comparison of chronological age (CA): Australian (AUS) and United Kingdom (UK)

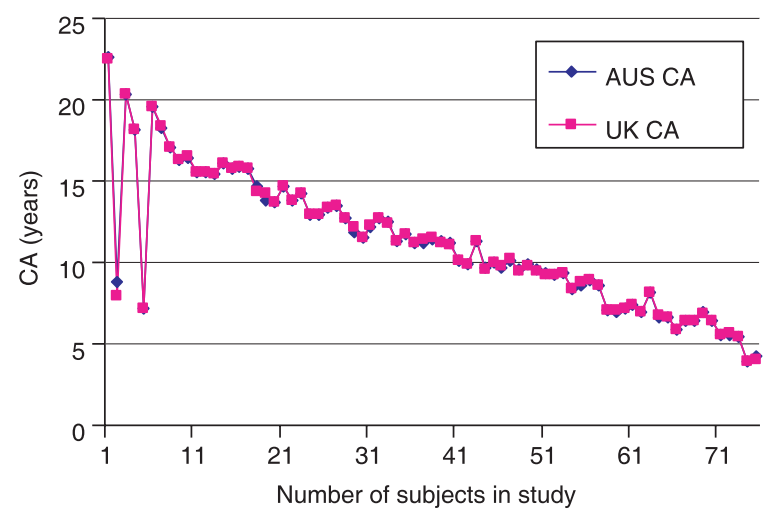

Fig. 4. Comparison of Australian (AUS) and United Kingdom (UK) chronological age (CA).

then averaged within subjects using the mathematical techniques of meta-analysis ${ }^{22}$.

The standards used in this study were derived from a British population. Male and female subjects were assessed using the same standards. At the time of the study, the British population was not partitioned by ethnicity, hence the reference data should not be mistaken for a homogenous population.

As UK and AUS populations have not previously been compared using a combination of Demirjian's anatomical stages ${ }^{1}$ and numerical data from a meta-analysis of a single subject ${ }^{22}$,

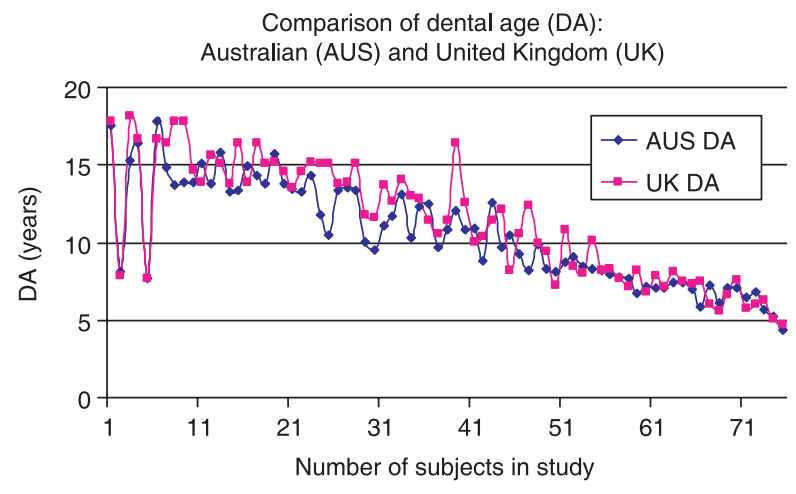

Fig. 5. Comparison of Australian (AUS) and United Kingdom (UK) dental age (DA).

a number of new findings came to light from the results of this study.

The CA and DA of the UK subjects were compared. The two variables were found to be similar. This result provides strong support for the estimation of DA using the mathematical techniques of meta-analysis.

The need for Demirjian's DAA method to be used in a test population using a reference data set from the same population has been addressed in a number of previous studies. This study, however, did not use Demirjian's full method; it only used the anatomical descriptions of TDS. The timing of dental development may not be the same in different 
population groups ${ }^{6}$, hence adjustments in developmental standards of one population may need to be made when used in another population ${ }^{6}$. This should improve the accuracy when determining CA.

In a previous study, children aged between 4 and 9 years of different ethnic groups living in London were compared using the full Demirjian method ${ }^{14}$. The aim was to determine ethnic differences in tooth formation. No difference in permanent tooth formation was demonstrated between Bangladeshi and white Caucasian children ${ }^{14}$. This indicates that the need to use a reference data set of the same population as the population being studied may not be required in DAAs. Previous studies are divided on this issue as some show positive results, whereas others show vast differences, the latter perhaps being attributed to a lack of discrimination of the method used.

The findings of this study show that using a specific population as the reference data set gives accurate determination of $\mathrm{CA}$ for that group using this new DAA method. This is demonstrated in the UK subjects.

When analysing the AUS population, the CA and DA were compared. In this study, these two variables were found to be significantly different $(P<0.001)$. In a DAA, such a discrepancy is used to indicate how advanced or delayed, on average, a sample population's DA is ${ }^{24}$. In this study, this discrepancy may be explained by the study using a reference data set which is clearly different from the population of subjects studied.

A DAA of a homogenous population should be assessed using scales that were devised on the same population ${ }^{5}$. This requirement is to ensure accuracy when converting results of a DAA to DA. At present, no AUS data reference set is as yet established, hence it is not possible to perform a DAA using an AUS reference set. The AUS population as such is culturally diverse. The sample used in this study is multicultural and, hence, representative of the vast array of cultural backgrounds in Australia. To be able to have a reliable data reference set, an excess of 4000 DPTs must be collected. This number of radiographs will ensure in excess of 100 data points for each TDS ensuring a sufficiently large reference data set.
Approximately 21 of the 77 AUS subjects had a DA of greater than 1 year compared to the CA. The discrepancy noted in this study is initially identified as a weakness; however, the result is consistent with using a different population reference data set to the subjects being tested. As discovered in previous DAA studies, the timing of TDS may differ in different population groups ${ }^{6}$. The difference in estimated dental and CA is hence plausible and explicable in this study.

The CA of the AUS and UK populations was compared. There were no significant differences noted. This is because the UK subjects were age $( \pm 0.25$ years $)$ and gender matched to the AUS subjects (see 'Identifying appropriate UK controls' in Methods section).

The two diverse populations were also compared with regard to DA and CA. It was found that the AUS population DA was 0.82 years or 9.84 months behind the UK DA. This result was highly significant $(P<0.001)$. This finding could be caused by the following reasons. Firstly, it suggests that AUS subjects aged between 4 and 24 years of age are approximately 9 months delayed in their dental development when compared to the UK subjects. Secondly, this delay may be attributed to a number of factors including those of an environmental, genetic, and social nature. Previous DAA studies have demonstrated different populations to have different rates of dental development. Black African children were shown to have earlier tooth emergence into the oral cavity than comparable Indian or Caucasian children ${ }^{25}$. Another dental maturity study found Australians to have the fastest dental maturation, whereas Korean children had the slowest dental maturation ${ }^{26}$. The different rates of dental development in different ethnic groups make the delay in dental development demonstrated in this study explicable.

It is hypothesized that using an AUS data set will diminish any discrepancies between DA and CA. Further analysis and creation of an AUS data reference set are essential to determine the reliability of the result of this study.

The sample size in this study is limited, but it provides a satisfactory starting point for further research. A sample size in the hundreds to thousands for the reference data set is an optimal number for assessment of DAA accuracy in different populations. 
Further, it must be noted that both the AUS and UK populations are multicultural. Hence, sample homogeneity is affected by the ethnic mix of subjects within each population. This has a bias on the results which cannot be further explained without further research.

In conclusion, the new method of DAA used in this study is seen as a reliable indicator of CA in UK children aged 4-24 years when using a UK reference data set. The actual method of using Demirjian's anatomical drawings of the TDS to assess tooth mineralization on a DPT is also reliable. The 9-month difference in dental development in the AUS population strongly indicates the limitations in using a UK reference data set to determine AUS DA. Further research and generation of a reference data set specific to the AUS population are needed. The clinical importance of such findings contributes to the determination of age, in disciplines within clinical dentistry, forensic medicine, and social deliberations.

What this paper adds

- The Demirjian method of DAA is seen as a reliable indicator of CA of UK children aged 4-24 years.

- The assessment of dental development of culturally diverse populations is limited by the use of a different reference set to the population involved, sample homeogenity, and social and environmental factors.

- Further research is essential to eludicate the findings of the delay in dental development found in the AUS population.

Why this paper is important to paediatric dentists

- DAA based on TDS is a reliable method in the determination of the CA of children of unknown birth date.

- Determination of age is of utmost importance in disciplines including paediatric clinical practice, forensic dentistry, and in social situations where age determination is paramount in legal proceedings.

\section{Acknowledgement}

This study was supported by an Undergraduate Research Grant 2006/2007 awarded by the Australian Dental Research Foundation.

\section{References}

1 Demirjian A, Goldstein H, Tanner JM. A new system of dental age assessment. Hum Biol 1973; 45: 211-227.
2 Moorrees CFA, Fanning EA, Hunt EH Jr. Age variation of formation stages for ten permanent teeth. $J$ Dent Res 1963; 42: 1490-1502.

3 Nystrom M, Aine L, Peck L, Haavikko K, Kataja M. Dental maturity in Finns and the problem of missing teeth. Acta Odontol Scand 2000; 38: 49-56.

4 Demirjian A. Interrelationships among measures of somatic, skeletal, dental and sexual maturity. Am J Orthod 1985; 88: 433-438.

5 Koshy S, Tandon S. Dental age assessment: the applicability of Demirjian's method in South Indian children. Forensic Sci Int 1998; 94: 73-85.

6 Nykanen R, Espeland L, Kvaal SI, Krogstad O. Validity of the Demirjian method for dental age estimation when applied to Norwegian children. Acta Odontol Scand 1998; 36: 238-244.

7 Maber M, Liversidge HM, Hector MP. Accuracy of age estimation of radiographic methods using developing teeth. Forensic Sci Int 2006; 159S: S68-S73.

8 Nolla CM. The development of permanent teeth. $J$ Dent Child 1960; 27: 254-266.

9 Gleiser I, Hunt EE. The permanent mandibular first molar; its calcification, eruption and decay. Am J Phys Anthropol 1955; 13: 253-284.

10 Harris MJP, Nortje CJ. The mesial root of the third mandibular molar. A possible indicator of age. $J$ Forensic Odontostomatol 1984; 2: 39-43.

11 Kullman L, Johanson G, Akesson L. Root development of the lower third molar and its relation to chronological age. Swed Dent J 1992; 16: 161167.

12 Gustafson G, Koch G. Age estimation up to 16 years of age based on dental development. Odontol Revy 1974; 25: 297-306.

13 Olze A, Bilang D, Schmidt S, Wernecke K-D, Geserick G, Schmeling A. Validation of common classification systems for assessing the mineralization of third molars. Int J Legal Med 2005; 119: 22-26.

14 Liversidge HM, Speechly T. Growth of permanent mandibular teeth of British children aged 4 to 9 years. Ann Hum Biol 2001; 28: 256-262.

15 Demirjian A, Goldstein H. New systems for dental maturity based on seven and four teeth. Ann Hum Biol 1976; 3: 411-421.

16 Eid RMR, Simi R, Friggi MNP, Fisberg M. Assessment of dental maturity of Brazilian children aged 6 to 14 years using Demirjian's method. Int J Paediatr Dent 2002; 12: 423-428.

17 Liversidge HM, Lyons F, Hector MP. The accuracy of three methods of age estimation using radiographic measurements of developing teeth. Forensic Sci Int 2003; 131: 22-29.

18 McKenna CJ, James H, Tayor JA, Townsend GC. Tooth development standards for South Australia. Aust Dent J 2002; 47: 223-227.

19 Farah CS, Booth DR, Knott SC. Dental maturity of children in Perth, Western Australia, and its application in forensic age estimation. J Clin Forensic Med 1999; 6: 14-18. 
20 Liversidge HM, Speechly T, Hector MP. Dental maturation in British children: are Demirjian's standards applicable? Int J Paediatr Dent 1999; 9: 263-269.

21 Liversidge HM, Chaillet N, Mornstad N, et al. Timing of Demirjian's tooth formation stages. Ann Hum Biol 2006; 33: 454-470.

22 Roberts GJ, Parekh S, Petrie A, Lucas VS. Dental age assessment (DAA): a simple method for children and emerging adults. Br Dent $J$ 2008; 204: E7.

23 Landis JR, Kosh GG. Measurement of observor agreement for categorical data. Biometric 1977; 33: 159174.
24 Nystrom M, Kleemola-Kujala E, Evalahti M, Peck L, Kataja M. Emergence of permanent teeth and dental age in a series of Finns. Acta Odontol Scand 2001; 59: 49-56.

25 Mugonzibwa EA, Kuijpers-Jagtman AM, Laine-Alava MT, van't Hof MA. Emergence of permanent teeth in Tanzanian children. Community Dent Oral Epidemiol 2002; 30: 455-462.

26 Chaillet N, Nyström M, Demirjian A. Comparison of dental maturity in children of different ethnic origins: international maturity curves for clinicians. J Forensic Sci 2005; 50: 1164-1174. 